\title{
Glioma stem cells are more aggressive in recurrent tumors with malignant progression than in the primary tumor, and both can be maintained long-term in vitro
}

\author{
Qiang Huang1, Quan-Bin Zhang2, Jun Dong*1, Yin-Yan Wu1 ${ }^{1}$, Yun- \\ Tian Shen ${ }^{3}$, Yao-Dong Zhao', Yu-De Zhu ${ }^{1}$, Yi Diao ${ }^{1}$, Ai-Dong Wang ${ }^{1}$ and \\ Qing Lan ${ }^{1}$
}

Address: ${ }^{1}$ Department of Neurosurgery and Brain Tumor Research Laboratory, the Second Affiliated Hospital of Suzhou University, Suzhou 215004, PR China, '2Department of Neurosurgery, Kowloon Hospital, Shanghai Jiaotong University Medical School, Suzhou 215021, PR China and ${ }^{3}$ Departments of Radiation Oncology, the Second Affiliated Hospital of Suzhou University, Suzhou 215004, PR China

Email: Qiang Huang - hq1936@yahoo.com.cn; Quan-Bin Zhang - quanbinzhang@yahoo.com.cn; Jun Dong* - djdongjun@163.com; YinYanWu - wchywyy@yahoo.com.cn; Yun-Tian Shen - shenyuntian2001@yahoo.com.cn; Yao-Dong Zhao - dm0920@gmail.com; YuDe Zhu - zyddzy2000@yahoo.com.cn; Yi Diao - dpdy118@yahoo.com.cn; Ai-Dong Wang - eagle.wangad@yahoo.com.cn;

Qing Lan - szlq006@yahoo.com.cn

* Corresponding author

Published: 22 October 2008

BMC Cancer 2008, 8:304 doi:10.1 |86/147|-2407-8-304
Received: 23 April 2008

Accepted: 22 October 2008

This article is available from: http://www.biomedcentral.com/I47I-2407/8/304

(C) 2008 Huang et al; licensee BioMed Central Ltd.

This is an Open Access article distributed under the terms of the Creative Commons Attribution License (http://creativecommons.org/licenses/by/2.0), which permits unrestricted use, distribution, and reproduction in any medium, provided the original work is properly cited.

\begin{abstract}
Background: Despite the advances made during decades of research, the mechanisms by which glioma is initiated and established remain elusive. The discovery of glioma stem cells (GSCs) may help to elucidate the processes of gliomagenesis with respect to their phenotype, differentiation and tumorigenic capacity during initiation and progression. Research on GSCs is still in its infancy, so no definitive conclusions about their role can yet be drawn. To understand the biology of GSCs fully, it is highly desirable to establish permanent and biologically stable GSC lines.

Methods: In the current study, GSCs were isolated from surgical specimens of primary and recurrent glioma in a patient whose malignancy had progressed during the previous six months. The GSCs were cryopreserved and resuscitated periodically during long-term maintenance to establish glioma stem/progenitor cell (GSPC) lines, which were characterized by immunofluorescence, flow cytometry and transmission electronic microscopy. The primary and recurrent GSPC lines were also compared in terms of in vivo tumorigenicity and invasiveness. Molecular genetic differences between the two lines were identified by array-based comparative genomic hybridization and further validated by real-time PCR.

Results: Two GSPC lines, SU-I (primary) and SU-2 (recurrent), were maintained in vitro for more than 44 months and 38 months respectively. Generally, the potentials for proliferation, self-renewal and multi-differentiation remained relatively stable even after a prolonged series of alternating episodes of cryopreservation and resuscitation. Intracranial transplantation of SU-I cells produced relatively less invasive tumor mass in athymic nude mice, while SU-2 cells led to much more diffuse and aggressive lesions strikingly recapitulated their original tumors. Neither SU-I nor SU-2 cells reached the terminal differentiation stage under conditions that would induce terminal differentiation in neural stem cells. The differentiation of most of the tumor cells seemed to be
\end{abstract}


blocked at the progenitor cell phase: most of them expressed nestin but only a few co-expressed differentiation markers. Transmission electron microscopy showed that GSCs were at a primitive stage of differentiation with low autophagic activity. Array-based comparative genomic hybridization revealed genetic alterations common to both SU-I and SU-2, including amplification of the oncogene EGFR and deletion of the tumor suppressor PTEN, while some genetic alterations such as amplification of MTAI (metastasis associated gene I) only occurred in SU-2.

Conclusion: The GSPC lines SU-I and SU-2 faithfully retained the characteristics of their original tumors and provide a reliable resource for investigating the mechanisms of formation and recurrence of human gliomas with progressive malignancy. Such investigations may eventually have major impacts on the understanding and treatment of gliomas.

\section{Background}

Recent decades have seen only limited progress in treatment trials and basic research on human glioma, the most common central nervous malignancy. This is partly because previously-established glioma cell lines are composed of morphologically and functionally diverse cells that express a variety of neural lineage markers [1]. It is now generally accepted that these previously-established serum-based cell lines do not replicate the major biological features, particularly the stem cells, of human cancers. Therefore, there is an urgent need for new and clinically relevant in vitro model systems for studying tumor biology and conducting preclinical screening of drugs for malignant brain tumors.

There is overwhelming evidence that glioma tissue contains stem cells that are broadly similar to neural stem cells but differ from them in important ways [2-5]. Although CD133, a 120 kDa cell-surface marker of normal human neural stem cells (NSCs), is not a specific marker or gold standard for identifying glioma stem cells, it has been used in most relevant studies for enriching tumor stem-like cells from brain tumors. Vescovi offered a functional definition of brain tumor stem cells, namely: brain tumor cells should qualify as stem cells if they show cancer-initiating ability upon orthotopic implantation, extensive self-renewal ability demonstrated either ex vivo or in vivo, karyotypic or genetic alterations, aberrant differentiation properties, capacity to generate non-tumorigenic end cells, and multilineage differentiation capacity [6]. Because this subpopulation of glioma cells, generally called glioma stem cells (GSCs), may play an extremely critical role in the initiation and recurrence of gliomas, studies focusing on GSCs have been promoted rapidly. However, conclusions about the biological features of GSCs are not always consistent and sometimes even confusing [1,7-14]. Most investigators believe that short-term cultured stem cells are superior to those maintained longterm. However, since glioma tissues are in very short supply, it is difficult to find tumor stem cells readily for either biological or preclinical drug studies. Therefore, perma- nent GSC lines could serve such purposes better than GSCs maintained short-term.

Tumor recurrence is the primary cause of treatment failure and death in glioma patients. Although cancer stem cells are now widely believed to be responsible for tumor recurrence, we do not know whether such cells are exactly the same in recurrent tumors as in the primary tumor in cases of malignancy progression.

We have isolated GSCs from surgical specimens of both primary and recurrent gliomas in recent years. Fortunately, GSCs from primary and recurrent tumors in the same patient were screened out and could be maintained long-term [15]. In the current study, we describe the results of long-term (more than three years) in vitro growth of these GSCs and their detailed characterization. To ensure that GSCs are available for use when required, the cells were periodically cryopreserved and resuscitated during long-term culture and tested for their capacity to form new nonadherent tumor spheres upon retrieval. We also compared the biological characteristics of GSCs derived from the primary and recurrent tumors in a patient with malignancy progression.

\section{Methods \\ Clinical Information}

Our studies were approved by the Medical Review Board of Suzhou University Medical School. Signed informed consent was obtained from the patients or their legal guardians prior to sample acquisition. Fresh surgical specimens were obtained from a 52-year-old female patient who had undergone two operations with an interval of 6 months because of the rapid re-growth of tumor mass in the right temporal lobe. The patient had received one dose of radiotherapy (45 Gy) one month after the initial surgery, then three doses of chemotherapy (VM26 0.1 ivgtt $\times$ 3 days followed by MeCCNU $200 \mathrm{mg}$ PO once) at onemonth intervals. In recurrence, a new solitary focus emerged in the ipisilateral frontal lobe in addition to tumor re-growth in situ. The primary lesion was patholog- 
ically diagnosed as mixed glioma comprising anaplastic ependymoma and astrocytoma (WHO grade III), while the recurrent lesion had transformed and progressed into glioblastoma multiforme (WHO grade IV) (Fig. 1) despite the continuous post-surgical radiotherapy and chemotherapy.

\section{Isolation and culture of glioma stem cells}

Tumor tissues were washed and minced with fine scissors into small fragments. Single tumor cells and small clumps (3-5 cells per clump) were collected with a $35 \mu \mathrm{m}$ cell strainer, then resuspended in DMEM-F-12 culture medium (Gibco-Invitrogen) to achieve a final concentration of $1 \times 10^{8}$ live cells per $\mathrm{ml}$ as assessed by Trypan blue staining [16]. Tumor spheres were cultured as described previously with some modifications [15].

\section{Flow cytometry and FACS of CDI33-positive cells}

A single cell-suspension from the tumor spheres was centrifuged, and magnetic cell separation and fluorescence-activated cell sorting were performed as follows. The cells were dissociated and resuspended in PBS. For magnetic labeling, CD133/1 Micro Beads were used (Miltenyi Biotech). Positive magnetic cell separation (MACS) was carried out using several MACS columns in series according to the manufacturer's instructions (Miltenyi Biotec). Cells were labeled with phycoerythin (PE)-conjugated monoclonal antibodies against human CD133 (CD133/2-PE, Miltenyi Biotec) or isotype control antibody (IgG2b(mouse)-PE, Caltag Laboratories) and analyzed using a BD FACS Calibur. The isolated CD133+ cells were suspended in defined stem cell growth medium containing DMEM-F-12, $\mathrm{N}_{2}$ supplement (Gibco), penicillin G, streptomycin sulfate, recombinant human FGF-2 $(20 \mathrm{ng} /$ $\mathrm{ml}$; R\&D System) and recombinant human EGF $(20 \mathrm{ng} / \mathrm{ml}$; $R \& D$ Systems), and were plated at a density of $2 \times 10^{6}$ live cells per $75 \mathrm{~cm}^{2}$ on an uncoated plastic flask.
Aliquots of CD133+ tumor cells obtained from MACS were further flow-sorted by FACS. The cells were labeled with CD133/2-PE at $4^{\circ} \mathrm{C}$ for $10 \mathrm{~min}$ following the manufacturer's instructions, then washed and resuspended in stem cell growth medium; negative controls were performed with IgG2b(mouse)-PE antibody as recommended by the manufacturer. The cells were then flowsorted and dead cells were excluded by propidium iodide (PI) staining.

\section{Subsphere formation}

Tumor spheres of different passages were dissociated into single cells and plated at a density of one cell per well. Half the volume of each culture medium $(50 \mu \mathrm{l})$ was changed every three days. The formation of tumor spheres was observed under a phase-contrast microscope.

\section{Proliferation}

For proliferation assays, cells were plated on 96-well plates and cultured in $200 \mu \mathrm{l}$ stem cell growth medium/ well at a density of 2000 cells $/ \mathrm{ml}$. From day 0 to day 10 after plating, viable cells were quantified using the 3-(4,5dimethylthiazol-2-yl)-2, 5-diphenyltetrazolium bromidebased Colorimetric Assay Cell Proliferation kit (Roche) according to the manufacturer's instructions.

\section{Tumor spheres cultured alternately in serum-based medium and serum-free stem cell growth medium} Nonadherent tumor spheres were seeded into DMEM-F12 medium supplemented with $10 \%$ fetal bovine serum (FBS, Hyclone). After the spheres had attached to the bottom of the flask and grown into cell monolayers for two weeks, the cells were washed with PBS to remove fetal bovine serum and transferred to defined stem cell growth medium. These procedures were repeated and morphological changes in the tumor cells were observed under a phase-contrast microscope.

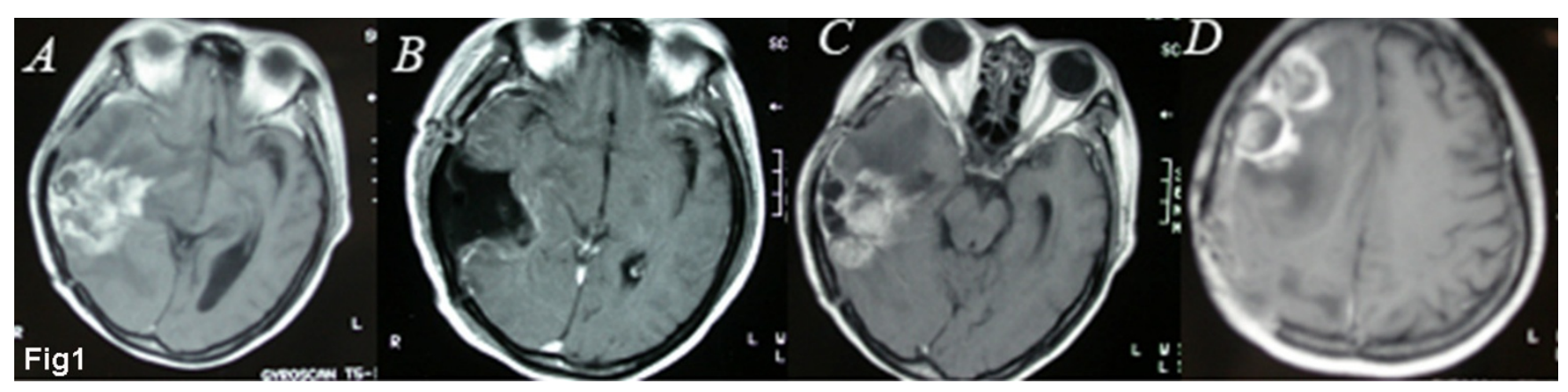

Figure I

MRI images of a 52-year-old female patient who underwent two operations with an interval of 6 months because of the rapid relapse of her tumor. A, The primary tumor located in the right temporal lobe and pathologically diagnosed as mixed glioma composed of anaplastic ependymoma and astrocytoma (WHO grade III). B, Post-operation image showing nearly total resection of the tumor. C, D, Relapse occurring 6 months later in situ and the ipsilateral frontal lobe; the recurrent lesion had transformed and progressed into glioblastoma multiforme (WHO grade IV). 


\section{Immunofluoresence staining to detect the expression of differentiation markers}

Isolated CD133+ cells were cultured in the aforementioned stem cell growth medium to allow tumor spheres to form. To determine the capacity of CD133+ cells for multi-lineage differentiation, the tumor spheres were transferred to poly-D-lysine coated chamber slides and cultured in DMEM-F12 supplemented with 10\% FBS. For immunofluorescence staining at different times during differentiation, namely days 0 (cultured for $4 \mathrm{~h}$ in serumbased medium), 3, 7 and 10, cells grown on the slides were fixed with $4 \%$ paraformaldehyde and permeabilized with $0.1 \%$ Triton X-100. The slides were then probed with mouse antibodies against human CD133, nestin (BD Bioscience), GFAP (Santa Cruz) and $\beta$-Tubulin-III (BD Bioscience). The secondary antibodies were either FITC- or Texas Red-conjugated anti-mouse IgG (Vector Laboratories). The cells were counterstained with 4',6'-diamidino2-phenylindole (DAPI; Vector Labs). Expression and/or coexpression of the aforementioned cell surface markers during differentiation was detected with a laser scanning confocal microscope (Carl Zeiss), and images were captured on a color CCD at specific magnifications.

\section{Tumorigenicity of GSCs}

Tumor cells from both SU-1 and SU-2 spheres were collected and suspended in $2 \mu \mathrm{l}$ PBS, then injected intracranially into the right caudate nucleus of athymic (NCR nu/ nu) mice. All procedures were conducted in accordance with Chinese laws governing animal care. Briefly, under the guidance of a stereotactic system, $2 \mu$ cell suspension $\left(1 \times 10^{8}\right.$ cells $\left./ \mathrm{ml}\right)$ in PBS were delivered into the right caudate nucleus $(0.2 \mu \mathrm{l} / \mathrm{min})$ by injection through a glass electrode connected to a Hamilton syringe. Mice were sacrificed when they became moribund or showed signs of obvious neurological deficit. Tumor samples were snapfrozen and frozen sections $(10 \mu \mathrm{m})$ were stained with HE following standard protocols.

\section{Transmission electron microscopy}

A single cell suspension from the tumor spheres was centrifuged, and magnetic cell separation and fluorescenceactivated cell sorting were performed to collect CD133+ GSCs. Positive cells were resuspended in PBS and the suspension was fixed in 4\% buffered glutaraldehyde, dehydrated through a graded ethanol series, embedded in Epon and cut into thin sections. The samples were imaged by a transmission electron microscope.

\section{Array-based comparative genomic hybridization (array- CGH)}

Genome-wide array comparative genomic hybridization was performed to determine changes in the copy number of genomic DNA from SU-1 and SU-2 GSCs. The array used in this study consisted of 2632 human BACs (Spec- tral Genomics, Houston, TX) spaced at approximately 1 megabase $(\mathrm{Mb})$ intervals across the whole genome. The experiments were performed according to the manufacturer's protocol, as described previously [17]. Briefly, the arrays were pre-hybridized with human Cot-I DNA (Gibco Invitrogen, Carlsbad, CA) and salmon testis DNA to block the repetitive sequences on BACs. One microgram of normal DNA (reference) or tumor DNA (test) was labeled with cy5-dUTP and cy3-dUTP by random priming. To avoid dye bias, we performed dye swap experiments for each sample. The probe mixture was dissolved in hybridization mixture, denatured, cooled and mounted with a $22 \times 60 \mathrm{~mm}$ coverslip. Hybridizations were performed in sealed chambers for $16-20 \mathrm{~h}$ at $60^{\circ} \mathrm{C}$. After post-hybridization washes, the arrays were scanned into two 16-bit TIFF image files using a GenePix 4000A twocolor fluorescent scanner and quantified using GenePix software (Axon Instruments, Union City, CA). Data were analyzed using SpectralWare 2.2.23 (Spectral Genomics, Houston, TX).

\section{Quantitative real-time PCR}

Real-time PCR was performed using SYBR green reagent and the ABI PRISM 7000 Sequence Detection System (PE Applied Biosystems) according to the manufacturer's instructions. $\beta$-actin transcripts were quantified in all samples as an internal control for the amount and quality of cDNA. Detailed information about primer sequence and product size is available upon request. The primer sets were all optimized to generate a single specific band only from cDNA on argarose gels. Melting-curve analysis was performed for all the reactions to control for the specificity of amplifications. The results of real-time PCR were analyzed by the $\Delta \Delta \mathrm{Ct}$ method and presented as the ratio between the selected genes and $\beta$-actin transcripts. The selected gene/ $\beta$-actin ratio was then normalized to the mean ratio of the selected genes in normal peripheral blood (for DNA) or in cultured normal human astrocytes (Cambrex, East Rutherford, NJ) (for mRNA) to calculate the Tumor/Normal ratio. All experiments were performed in triplicate.

\section{Results \\ Growth and differentiation of GSCs maintained long-term in vitro}

Glioma stem/progenitor (GSPC) cell lines, named SU-1 for the primary tumor and SU-2 for the recurrent tumor associated with malignancy progression, were maintained in vitro for more than 44 months and 38 months, respectively. Both lines contained an obvious CD133+ subpopulation that displayed neurosphere-like tumor spheres, nonadherent growth and asymmetrical cell divisions yielding cells that expressed markers of neuron and glia differentiation. The expression and coexpression of differentiation-related surface makers were examined by laser 
scanning confocal microscopy. Within a single sphere grown from one CD133+ GSC, the cells expressed markers of different neural lineages at constant proportions; most of the tumor cells did not differentiate terminally and often co-expressed differentiation markers with the stem/ progenitor cell marker nestin. Over $90 \%$ of the cells in both SU-1 and SU-2 tumor spheres were nestin positive (Fig. 2A) and about 5-10\% stained positively for CD133 (Fig. 2B). When the tumor spheres became adherent mon- olayers, one week after FBS-induced differentiation without growth factor supplementation in vitro, about 78$83 \%$ of the cells were still nestin positive (Fig. 2D, 2G). However, about $17-22 \%$ of these nestin+ cells were also positive for such differentiation markers as GFAP for astroglial cells (Fig. 2E) and/or $\beta$-tubulin-III for neurons (Fig. $2 \mathrm{H}$ ), confirming that they retained multi-lineage differentiation capabilities. Therefore, GSCs cannot reach the stage of complete terminal differentiation under condi-
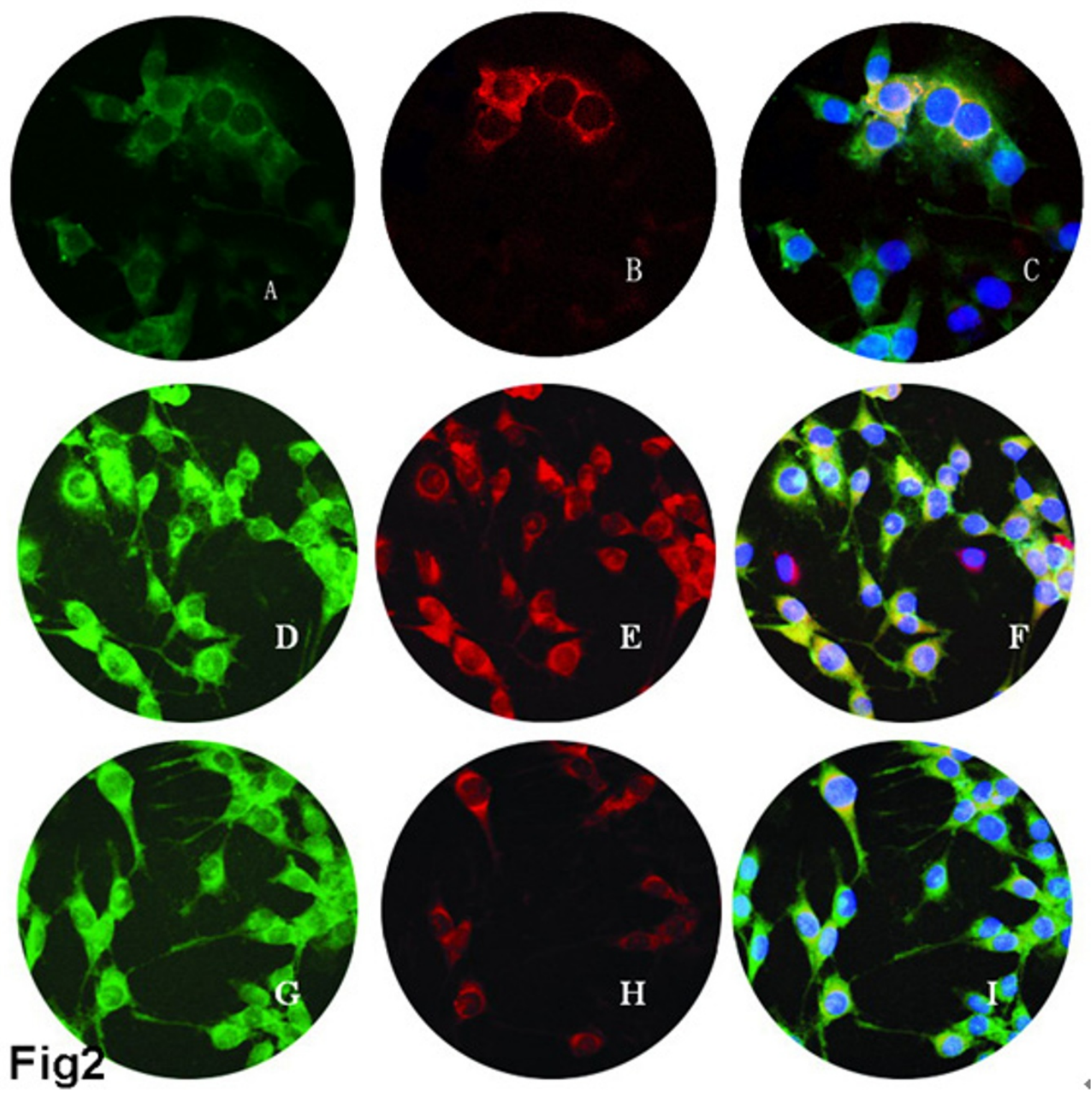

Figure 2

Expression of cell surface markers of tumor spheres before and after differentiation $(\times \mathbf{4 0 0})$. A-C, tumor spheres before differentiation. D-I, one week after FBS-induced differentiation. A, D, G, immunofluorescence staining for nestin (green). B, immunofluorescence staining for CDI33 (red). E, immunofluorescence staining for GFAP (red). H, immunofluorescence staining for $\beta$-tubulin-III (red). C, F, I, confocol microscopy showed co-expression of nestin with CDI33, GFAP and $\beta$ tubulin-III, respectively. 
tions that would induce terminal differentiation in NSCs. In vitro data suggest that the differentiation of most GSCs is blocked at the neural progenitor stage, as indicated by the expression of nestin, or at the early stage of tumor progenitor cell differentiation, as indicated by the partial coexpression of nestin and glial/neural differentiation markers.

Nonadherent tumor spheres were dissociated and magnetically sorted into CD133+ and CD133- cells. Upon replating at one cell per well, tumor spheres formed from single CD133+ cells, often reaching a size of 30-40 cells in approximately two weeks. Only a small proportion (4.5\% for SU-1 and $12.8 \%$ for SU-2) of the CD133+ tumor cells formed spheres, but no in vitro sphere formation was observed with CD133- cells. Sequential minimal dilution assays for three passages confirmed that the CD133+ single cell-derived tumor spheres had the potential to grow indefinitely. The proportion of sphere-form- ing cells remained stable throughout the course of culture, indicating asymmetrical cell divisions. Periodically, the tumor sphere cells were cryopreserved and resuscitated; the resuscitation rate was about $75 \%$. However, SU-1 and SU-2 showed some morphological differences in vitro. Briefly, when cultivated in defined stem cell growth medium (FBS free), the SU-1 tumor spheres were more compact while the SU-2 ones seemed a little looser and were not evenly round (Fig. 3A, 3D). When cultured in medium containing 10\% FBS, both SU-1 and SU-2 cells changed morphologically from rolling spheres to adherent monolayers. Most of the differentiated SU-1 cells were star- or spindle-shaped (Fig 3B), but most of the differentiated SU-2 cells were round or oval (Fig 3E). Fluorescence-activated cell sorting confirmed the presence of CD133+ cells. The relative content of CD133+ cells was markedly higher in the recurrent cell line SU-2 (8.1\%, Fig $3 \mathrm{~F})$ than in the primary tumor cell line SU-1 (2.0\%, Fig

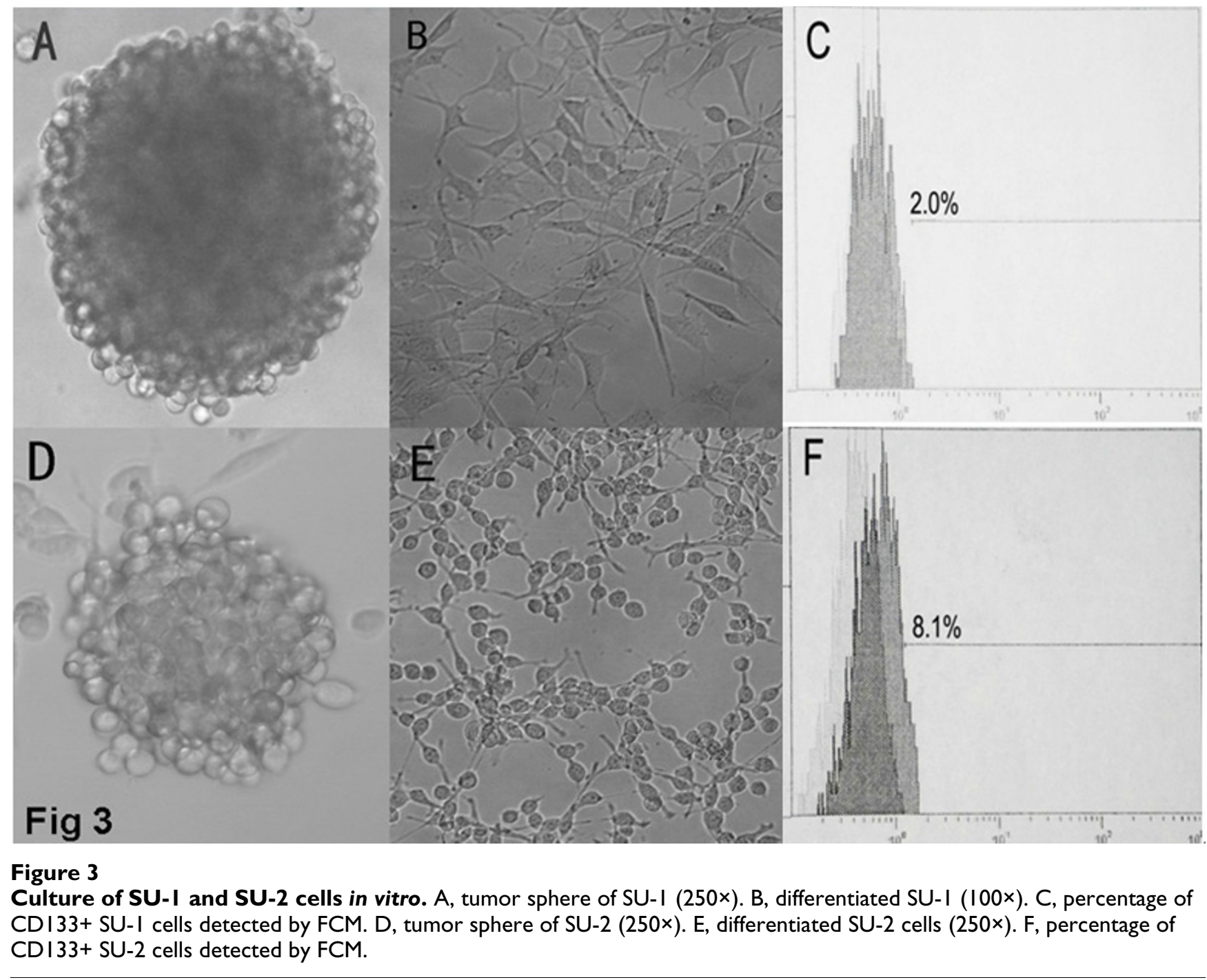


3C). Non-specific staining was $1.3 \%$ for SU-1 and $0.6 \%$ for SU-2.

\section{Intracranial xenografts derived from SU-2 are more aggressive than those from SU-I}

When inoculated intracranially into athymic (NCR nu/ nu) mice, both SU-1 and SU-2 reproducibly established tumors (5/5 for both SU- 1 and SU-2). The average median survival time was 32 days for SU-1 and 27 days for SU-2. Pathologically, SU-1 cells gave rise to less invasive tumor masses; most of the tumor was restricted to the ipsilateral hemisphere of the injection site, and nodular tumor formation was observed (Fig. 4A, 4B). In contrast, tumors derived from SU-2 cells showed diffuse infiltrating growth patterns; they were distinctive in that they were widely disseminated, invaded the ipsilateral hemisphere, migrated to the cortex on the contralateral side and infiltrated the subarachnoid space of the longitudinal fissure, recapitulating the original recurrent lesion (Fig. 4C). Moreover, most tumor cells were primitive round or oval, distributed evenly and densely without forming obvious tumor masses, morphologically resembling gliomatosis cerebri (Fig. 4D), indicating that the latter may be a stem cell disease. This possibility deserves further investigation.

\section{Transmission electron microscopy showed that GSCs from both SU-I and SU-2 lacked autophagosomes}

Examined by transmission electron microscopy, the nuclei of GSCs from SU-1 and SU-2 were large and regular, circular or oval in shape, with typical inner and outer nuclear membranes, nucleopores, perinuclear space and other structures. Euchromatin was far more abundant than heterochromatin. Two or more nucleoli were often observed with clear fibrillar centers (FC), dense fibrillar components (DFC) and granular components (GC). In the cytoplasm, ribosomes and mitochondria were abundant while the Golgi apparatus and endoplasmic reticulum were relatively less developed; lysosomes (including autophagosomes) were rare in comparison with NSCs.
Taken together, these ultrastructural features implied that the GSCs were at a primitive stage of differentiation with low autophagic activity (Fig. 5).

\section{Clones with altered DNA copy number identified by array- based CGH}

Among the 2621 clones spotted on the array, 2457 were mapped to autosomes. Changes in the DNA copy number were found in 160 clones of GSCs from SU-1 and 182 clones from SU-2. With increased resolution of the BAC array, regions with changes in DNA copy number spanning more than $1 \mathrm{Mb}$ could be detected in an average of two clones. The clustered changes were defined as regions with at least two consecutive adjacent clones within $1 \mathrm{Mb}$ on the linear map of the human genome sequence. A total of 7 clustered regions on chromosomes $1 \mathrm{p}, 2 \mathrm{p}, 2 \mathrm{q}, 4 \mathrm{q}, 9 \mathrm{p}$, $14 \mathrm{q}$ and $19 \mathrm{q}$ were identified in GSCs of both SU-1 and SU-2 (Additional file 1). Subsequent searching of the human genome draft revealed several prominent genes in these regions that have often been associated with human cancer, and these were also found to be altered (Additional file 1). Small gains or losses restricted to single BAC clones were also detected. Most of the altered clones were detected in GSCs from both SU-1 and SU-2. Among the isolated $\mathrm{BAC}$ clones in which the tumor/normal ratio exceeded 1.3 (gain) or was less than 0.75 (loss), a clone on $7 \mathrm{p} 12$ containing the gene for epidermal growth factor receptor $(E G F R)$ was amplified (Figure $6 \mathrm{~A})$. The clone RP11-129G17 on 10q23 had the lowest tumor/normal ratio. Since the tumor suppressor gene PTEN is mapped exactly to the region covered by RP11-129G17, this finding suggests that PTEN was deleted in GSCs of both the SU-1 and SU-2 lines, as has frequently been reported in gliomas (Figure 6B). These genetic changes included deletion of p18INK4 and $R b$ as well as the tumor suppressor $P T E N$, and amplification of the oncogenes CDK7 and EGFR. We also found some genetic changes that were exclusive to either SU-1 or SU-2. The most intriguing of these was the amplification of MTA 1 in SU-2 (metastasis

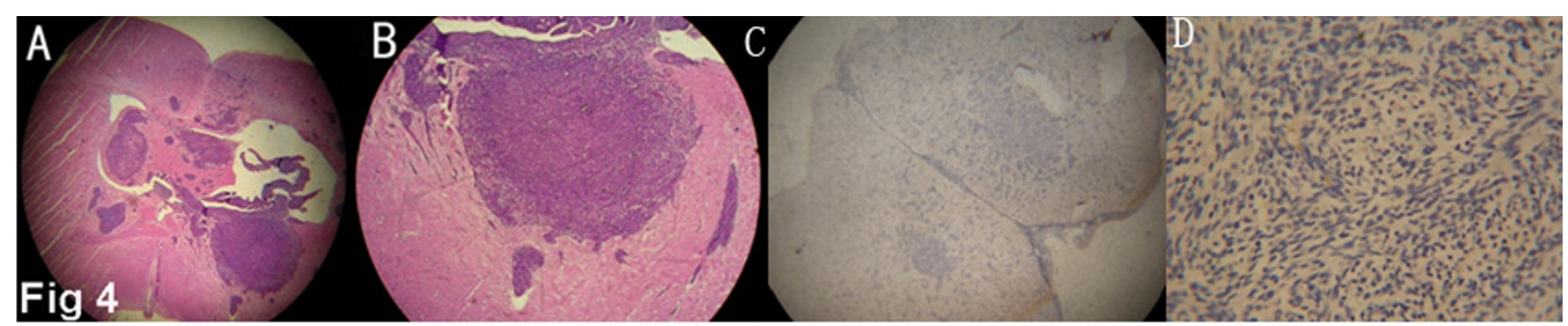

\section{Figure 4}

Pathological examination of intracranial tumor mass in NC nude mice. A, SU-I cells produced relatively well-delimited tumor masses $(\mathrm{HE} \times 20)$. B, local amplification of $A$, showing nodular tumor formation $(\mathrm{HE} \times 100)$. C, SU-2 cells behaved more aggressively $(\mathrm{HE} \times 20)$. D, SU-2 cells infiltrated remoter regions and invaded the whole brain evenly and densely $(\mathrm{HE} \times$ 200). 


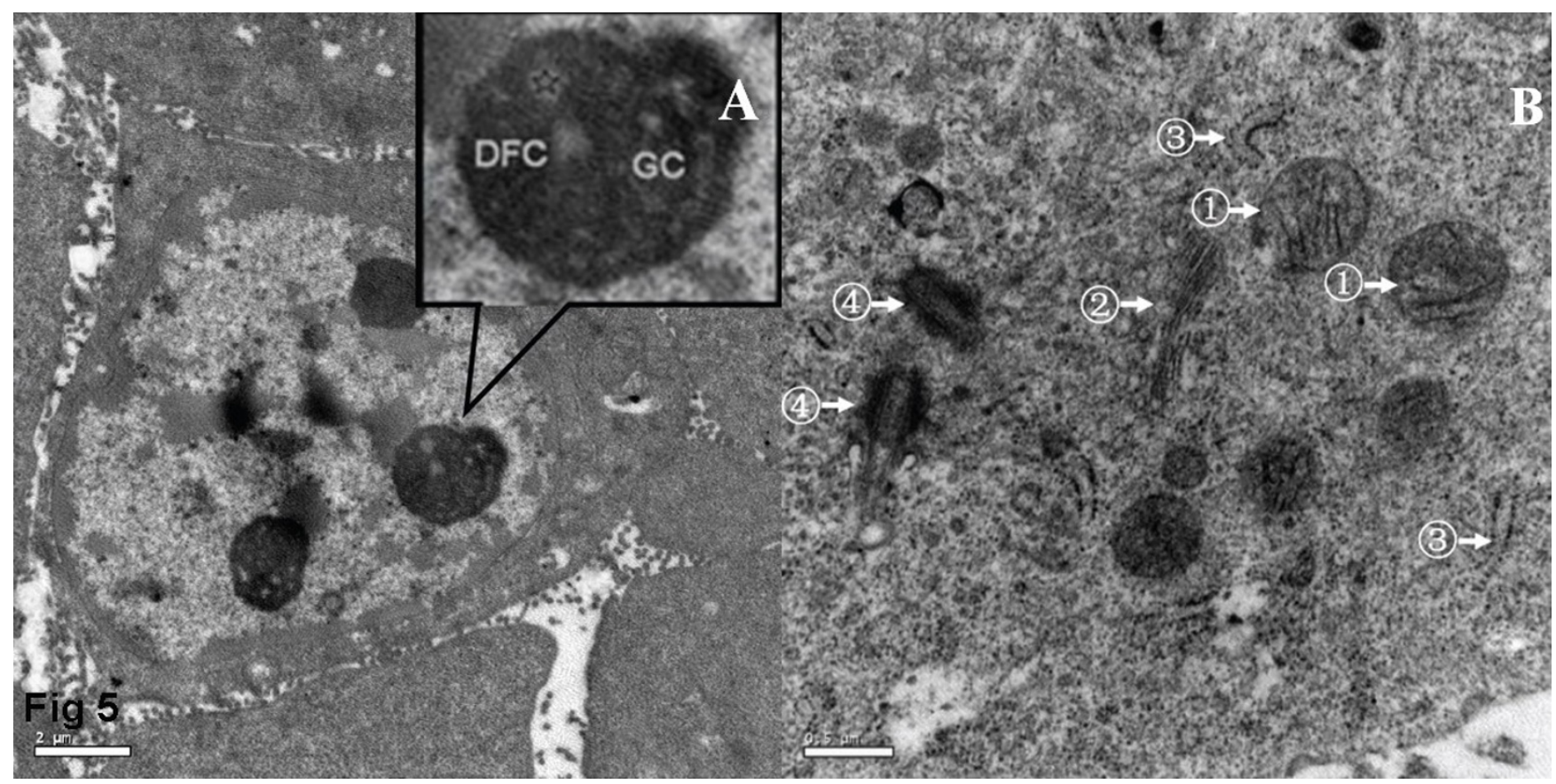

\section{Figure 5}

GSCs observed under transmission electron microscopy (TEM) showed a lack of autophagosomes. A, nuclei and chromatin, showing a high nucleus: cytoplasm ratio, large oval nucleus and nucleoli (the asterisk indicates the FC). B, mitochondria (arrow (1), Golgi apparatus (arrow (2), rough endoplasmic reticulum (arrow (3), a couple of centrioles (arrow (4)).

associated 1), a gene previously found to be closely related to the recurrence and metastasis of breast cancer, osteosarcoma and other malignances (Additional file 2).

\section{Real-time PCR validated the results of the array-based CGH}

To validate the array-based CGH findings, quantitative real-time PCR was performed to examine the expression of genes encoding EGFR, PTEN, CDC2 and DNMT3B. Consistent with the array-based CGH results, real-time PCR showed increased expression of EGFR and DNMT3B
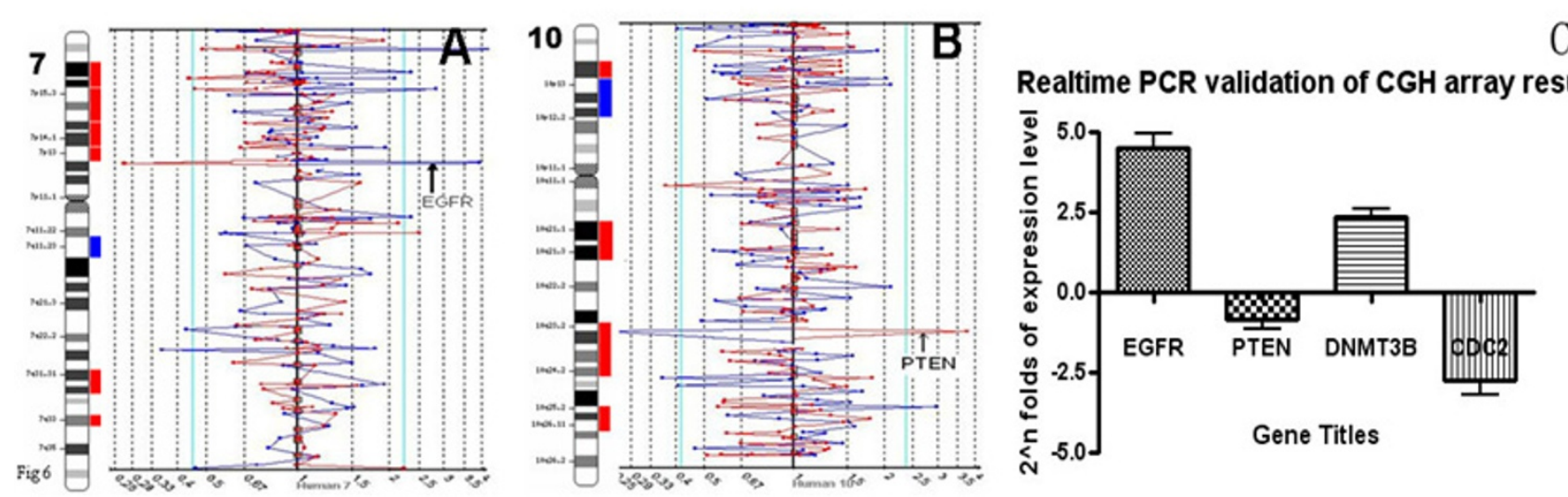

\section{Figure 6}

Copy number changes in genomic DNA from GSCs of both SU-I and SU-2 lines were identified by the CGH array, the ideograms show the gains (blue) and losses (red) of DNA copy numbers. Representative ratio plots of chromosomes 7, with amplification of oncogene EGFR (A), and 10, with deletion of tumor suppressor PTEN (B); Real-time quantitative $P C R$ validated some of array-based $C G H$ results $(C)$. 
and down-regulation of PTEN and CDC2 in GSCs compared to normal human NSCs (Figure 6C).

\section{Discussion}

In the past few years, stem cell-like tumor precursors have been identified in gliomas. They have been consecutively termed glioma stem cells, brain tumor stem cells or brain tumor initiating cells. They are characterized by selfrenewal, limitless proliferation, tumor initiation, multidifferentiation and expression of stem cell surface markers such as CD133 and nestin. However, long-term stable maintenance of GSCs, which will offer much more convenient opportunities for attaining full and accurate understanding of the biological features of this special tumor cell type, has been achieved by only a few groups and does not suffice to meet research requirements $[18,19]$. No pure CD133+ glioma stem cell line has so far been available; proliferation and differentiation of these tumor stem cells in vitro cannot be stopped completely even in a culture medium favoring stem cell growth. The percentage of $\mathrm{CD} 133+$ cells in such lines has varied widely. Accordingly, there are no unanimously agreed criteria for establishing a GSC line. Successful cell lines from other tumors suggest that establishment of a GSC line should meet the following criteria. First, GSCs can be cultured long-term in vitro while maintaining relatively stable stem cell properties. Secondly, even after long-term maintenance, the GSCs should recapitulate their parent or original tumor. In the current study, SU-1 and SU-2, respectively originating from primary and recurrent gliomas with malignancy progression in the same patient, have been maintained in vitro for more than three years while retaining their tumor stem cell properties. Though the percentage of CD133+ cells was not high (less than $10 \%)$, nestin+ cells were the dominant subgroup (>90\%). Thus, the two newly established cell lines SU-1 and SU-2 could be regarded as glioma stem/progenitor lines. Cryopreservation and resuscitation were successful during long-term serial passages in vitro. We also noticed differences in configuration between the tumor spheres derived from SU-1 and SU-2. When cultured in defined stem cell growth medium (FBS free), the SU-1 spheres were more compact than those of SU-2, and the percentage of CD133+ cells was lower. When cultured in serum-based medium, SU-2 seemed more resistant to FBS-induced differentiation and remained more morphologically primitive than SU-1. In vivo, direct orthotopic transplantation of SU-1 and SU-2 cells developed into xenografts in immune-deficient mouse cerebrum, but the tumors derived from SU-2 cells were more aggressive than those from SU-1. These data imply that malignancy progression could also occur in tumor stem cells. Taken together, these results suggest that SU- 1 and SU- 2 could provisionally be regarded as permanent glioma stem/progrnitor cell lines and further utilized as reliable resources for basic research and clinical trials concerning GSCs.

The study of GSCs is actually an extension of that of NSCs, since not only the concepts but also the methods employed are derived from those used for NSCs. The finding that $10^{2} \mathrm{CD} 133+$ tumor cells could produce tumor mass in NOD-SCID mice, while up to $10^{5} \mathrm{CD} 133$ - tumor cells could not, proved that the former were brain tumor initiating cells and the latter were not [8]. So it seemed reasonable to suppose that $\mathrm{CD} 133+$ tumor stem cells could proliferate and differentiate into CD133- cells, which could further differentiate into common tumor cells approaching terminal differentiation, as NSCs do. However, Beier's studies revealed that four of 15 cell lines derived from primary glioblastomas grew adherently in vitro and were driven by CD133- tumor cells that fulfilled stem cell criteria. Both CD133+ and CD133- subtypes of GSCs were similarly tumorigenic in nude mice in vivo [20], indicating that CD133 expression is not sufficient to identify GSCs; more effort is needed to identify a specific GSC marker. At present, though this functional criterion for GSCs is sophisticated and inconvenient to apply, it is reliable and should not be neglected unless and until a specific marker for GSCs is found.

GSCs do not differentiate terminally under conditions that would induce terminal differentiation in NSCs. Not only was differentiation retarded, but retro-differentiation was also observed in vitro. Our data showed that soon after treatment with differentiation-inducing agents such as FBS and valproate (VPA), nonadherent tumor spheres dissociated and scattered into adherent spindle-shaped monolayer cells. Most of these were still highly positive for nestin (a marker for neural stem/progenitor cells), while a few cells appeared that were doubly positive for nestin and either GFAP (marker for astrocytes) or $\beta$-tubulin III (marker for neurons). Markers of both mature and stem/ progenitor cells are very rarely co-expressed during NSC differentiation, but it is common in GSCs [15]. We also observed a "down-up" trend in the percentages of CD133+ cells in SU-1 and SU-2 during a relatively long differentiation-inducing process in vitro; that is, the percentage of CD133+ cells decreased at first, then remained low for a time and finally increased a little, suggesting that partially differentiated CD133+ cells (loss of CD133 expression) retro-differentiated into CD133+ GSCs under certain circumstances, which made the GSCs involved in tumor remodeling more sophisticated. There was a concomitant "up-down" trend in the levels of the neural differentiation markers GFAP and $\beta$-tubulin-III. These phenomena were more obvious in SU-2 [15]. Thus, it is easy to infer that GSCs were generally similar to NSCs but showed important differences. Under conditions in which differentiation would be induced in NSCs, GSCs showed 
an intrinsic potential to maintain their undifferentiated state or to resist differentiation and even tended to retrodifferentiate under certain circumstances. Once differentiation was initiated in NSCs, they were transformed step by step into various kinds of mature neural cells.

Amplification of the oncogene EGFR and deletion of the tumor suppressor PTEN have been identified as the critical genetic changes in the tumorigenesis of human GBMs or other types of glioma. However, few existing glioma cell lines harbor these genetic abnormalities [21-32]. The fact that GSCs of both the SU- 1 and SU-2 lines faithfully preserved the EGFR amplification and PTEN loss greatly enhances their utility in biological and preclinical studies of human gliomas. Recent studies have shown a close correlation between PTEN loss and low autophagic activiy [33]. We also found that PTEN loss and absence of autophagy were concurrent in both SU-1 and SU-2, and this may suggest potential targets for future molecular intervention. More intriguingly, we discovered amplification of MTA1 in SU-2 but not in SU-1. MTA1 is closely associated with various malignancies and its up-regulation always indicates tumor recurrence and metastasis [34-39]. However, the significance of MTA1 in the malignancy progression of gliomas has rarely been considered. In the current study, the particular amplification of MTA1 in GSCs derived from the recurrent tumor makes it reasonable to conjecture that MTA1 activation may contribute to both the aggression of GSCs and the malignancy progression of gliomas.

\section{Conclusion}

In summary, we successfully established two glioma stem/ progenitor cell lines from primary and recurrent tumors with malignancy progression obtained from the same patient. We discovered that GSCs in the recurrent tumor with malignancy progression were more aggressive than in the primary tumor, which suggests that tumor progression may be initiated early in tumor stem cells. We also demonstrated that direct isolation and long term maintenance of GSCs from freshly resected glioma tissues is a feasible approach for future biological studies of cancer stem cells and pre-clinical testing of novel therapeutic agents.

\section{Competing interests}

The authors declare that they have no competing interests.

\section{Authors' contributions}

QH participated in the study design, carried out most of the experiments and drafted the manuscript. QBZ carried out the immunoassays and participated in the critical revision of the manuscript. JD conceived of the study, participated in its design and coordination and participated in manuscript preparation. YYW participated in clinical data collection and critically revised the manuscript. YTS car- ried out the stem cell culture. YDZ performed the ultramicroscopy of the stem cells. YDZ carried out the flow cytometry assay. YD performed the animal experiments. ADW carried out the molecular genetic studies and the critical revision of the manuscript. QL participated in the coordination of the study and the critical revision of the manuscript. All authors read and approved the final manuscript.

\section{Consent}

Written informed consent was obtained from the patient for publication of this article and accompanying radiographic images.

\section{Additional material}

\section{Additional file 1}

Summary of clustered DNA copy number changes in GSCs detected by CGH array

Summary of clustered DNA copy number changes in GSCs detected by CGH array

Click here for file

[http://www.biomedcentral.com/content/supplementary/14712407-8-304-S1.doc]

\section{Additional file 2}

Click here for file

[http://www.biomedcentral.com/content/supplementary/14712407-8-304-S2.doc]

\section{Acknowledgements}

The authors thank Dr. Ping Feng for her technical support with flow cytometry and our neuropathologist professor Zhen-Yan Liu for his excellent work. This study is supported by the National Natural Science Foundation of China (No.306721 64; 3077224I) and the Natural Science Foundation of jiangsu Province, China (No. BK2007507).

\section{References}

I. Singh SK, Clarke ID, Terasaki M, Bonn VE, Hawkins C, Squire J, Dirks $\mathrm{PB}$ : Identification of a cancer stem cell in human brain tumors. Cancer Res 2003, 63(18):582I-8.

2. Reya T, Morrison SJ, Clarke MF, Weissman IL: Stem cells, cancer, and cancer stem cells. Nature 200I, 4I4(6859): I05-II.

3. Gilbertson RJ: Brain tumors provide new clues to the source of cancer stem cells: does oncology recapitulate ontogeny? Cell Cycle 2006, 5(2): I35-7.

4. Galderisi U, Cipollaro M, Giordano A: Stem cells and brain cancer. Cell Death Differ 2006, I 3(I):5-II.

5. Sanai N, Alvarez-Buylla A, Berger MS: Neural stem cells and the origin of gliomas. N Engl J Med 2005, 353(8):8I I-22.

6. Vescovi AL, Galli R, Reynolds BA: Brain tumor stem cells. Nat Rev Cancer 2006, 6(6):425-36.

7. Ignatova TN, Kukekov VG, Laywell ED, Suslov ON, Vrionis FD, Steinder DA: Human cortical glial tumors contain neural stemlike cells expressing astroglial and neuronal markers in vitro. Glia 2002, 39(3): 193-206.

8. Singh SK, Hawkins C, Clarke ID, Squire JA, Bayani J, Hide T, Henkelman RM, Cusimano MD, Dirks PB: Identification of human brain tumor initiating cells. Nature 2004, 432(70 I 5):396-40I. 
9. Galli R, Binda E, Orfanelli U, Cipelletti B, Gritti A, De Vitis S, Fiocco $R$, Foroni $C$, Dimeco F, Vescovi A: Isolation and characterization of tumorigenic stem-like neural precursors from human glioblastoma. Cancer Res 2004, 64(19):70 I I-2 I.

10. Kondo T, Setoguchi T, Taga T: Persistence of a small subpopulation of cancer stem-like cells in the $\mathbf{C 6}$ glioma cell line. Proc Natl Acad Sci USA 2004, I 0 I(3):78I-6.

II. Fomchenko El, Holland EC: Stem cell and brain cancer. Exp Cell Res 2005, 306(2):323-9.

12. Zheng X, Shen G, Yang X, Liu W: Most $C 6$ cells are cancer stem cells: evidence from clonal and population analyses. Cancer Res 2007, 67(8):369l-7.

13. Yuan X, Curtin J, Xiong Y, Liu G, Waschsmann-Hogiu S, Farkas DL, Black KL, Yu JS: Isolation of cancer stem cells from adult glioblastoma multiforme. Oncogene 2004, 23(58):9392-400.

14. Hemmati HD, Nakano I, Lazareff JA, Masterman-Smith M, Geschwind $\mathrm{DH}$, Bronner-Fraser M, Kornblum $\mathrm{HI}$ : Cancerous stem cells can arise from pediatric brain tumors. Proc Natl Acad Sci USA 2003, I00(25): $15178-83$.

15. Zhang QB, Ji XY, Huang Q, Dong J, Zhu YD, Lan Q: Differentiation profile of brain tumor stem cells: a comparative study with neural stem cells. Cell Res 2006, I6(I2):909-15.

16. Reynolds BA, Weiss S: Generation of neurons and astrocytes from isolated cells of the adult mammalian central nervous system. Science 1992, 255(5052): 1707-10.

17. Man TK, Lu XY, Jaeweon K, Perlaky L, Harris CP, Shah S, Ladanyi M, Gorlick R, Lau CC, Rao PH: Genome-wide array comparative genomic hybridization analysis reveals distinct amplifications in osteosarcoma. BMC Cancer 2004, 4:45-54

18. Inagaki A, Soeda A, Oka N, Kitajima H, Nakagawa J, Motohashi T, Kunisada T, Iwama T: Long-term maintenance of brain tumor stem cell properties under at non-adherent and adherent culture conditions. Biochem Biophys Res Commun 2007, 36 I(3):586-92.

19. Günther HS, Schmidt NO, Phillips HS, Kemming D, Kharbanda S, Soriano R, Modrusan Z, Meissner H, Westphal M, Lamszus K: Glioblastoma-derived stem cell-enriched cultures form distinct subgroups according to molecular and phenotypic criteria. Oncogene 2008, 27(20):2897-909.

20. Beier D, Hau P, Proescholdt M, Lohmeier A, Wischhusen J, Oefner PJ, Aigner L, Brawanski A, Bogdahn U, Beier CP: CDI33 (+) and CDI33 (-) glioblastoma-derived cancer stem cells show differential growth characteristics and molecular profiles. Cancer Res 2007, 67(9):4010-5.

21. Giannini C, Sarkaria JN, Saito A, Uhm JH, Galanis E, Carlson BL, Schroeder MA, James CD: Patient tumor EGFR and PDGFRA gene amplifications retained in an invasive intracranial xenograft model of glioblastoma multiforme. Neuro Oncol 2005, 7(2): 164-76.

22. Pandita A, Aldape KD, Zadeh G, Guha A, James CD: Contrasting in vivo and in vitro fates of glioblastoma cell subpopulations with amplified EGFR. Genes Chromosomes Cancer 2004, 39(I):29-36

23. Ekstrand AJ, James CD, Cavenee WK, Seliger B, Pettersson RF, Collins VP: Genes for epidermal growth factor receptor, transforming growth factor alpha, and epidermal growth factor and their expression in human gliomas in vivo. Cancer Res |99|, 5 | (8):2164-72.

24. Wong AJ, Bigner SH, Bigner DD, Kinzler KW, Hamilton SR, Vogelstein $B$ : Increased expression of the epidermal growth factor receptor gene in malignant gliomas is invariably associated with gene amplification. Proc Natl Acad Sci USA 1987, 84(19):6899-903.

25. Nathoo N, Goldlust S, Vogelbaum MA: Epidermal growth factor receptor antagonists: novel therapy for the treatment of high-grade gliomas. Neurosurgery 2004, 54(6): | 480-8.

26. Cappuzzo F: Erlotinib in gliomas: should selection be based on EGFR and Akt analyses? J Natl Cancer Inst 2005, 97( I 2):868-9.

27. Groszer M, Erickson R, Scripture-Adams DD, Dougherty JD, Le Belle J, Zack JA, Geschwind DH, Liu X, Kornblum HI, Wu H: PTEN negatively regulates neural stem cell self-renewal by modulating G0-G I cell cycle entry. Proc Natl Acad Sci USA 2006, I 03( I): I I -6.

28. Knobbe $C B$, Merlo A, Reifenberger G: PTEN signaling in gliomas. Neuro Oncol 2002, 4(3): 196-2II.
29. Sansal I, Sellers WR: The biology and clinical relevance of the PTEN tumor suppressor pathway. J Clin Oncol 2004, 22(14):2954-63.

30. Li J, Yen C, Liaw D, Podsypanina K, Bose S, Wang SI, Puc J, Miliaresis C, Rodgers L, McCombie R, Bigner SH, Giovanella BC, Ittmann M, Tycko B, Hibshoosh H, Wigler MH, Parsons R: PTEN, a putative protein tyrosine phosphatase gene mutated in human brain, breast, and prostate cancer. Science 1997, 275(5308): 1943-7.

3I. Teng DH, Hu R, Lin H, Davis T, lliev D, Frye C, Swedlund B, Hansen KL, Vinson VL, Gumpper KL, Ellis L, El-Naggar A, Frazier M, Jasser S, Langford LA, Lee J, Mills GB, Pershouse MA, Pollack RE, Tornos C, Troncoso P, Yung WK, Fujii G, Berson A, Steck PA: MMACI/PTEN mutations in primary tumor specimens and tumor cell lines. Cancer Res 1997, 57(23):5221-5.

32. Ishii N, Maier D, Merlo A, Tada M, Sawamura $Y$, Diserens AC, van Meir EG: Frequent co-alterations of TP53, pI6/CDKN2A, pIAARF, PTEN tumor suppressor genes in human glioma cell lines. Brain Pathol 1999, 9(3):469-79.

33. Gozuacik D, Kimchi $A$ : Autophagy as a cell death and tumor suppressor mechanism. Oncogene 2004, 23(16):289|-906.

34. Balasenthil S, Broaddus RR, Kumar R: Expression of metastasisassociated protein I (MTAI) in benign endometrium and endometrial adenocarcinomas. Hum Pathol 2006, 37(6):656-6I.

35. Gururaj AE, Singh RR, Rayala SK, Holm C, den Hollander P, Zhang H, Balasenthil S, Talukder AH, Landberg G, Kumar R: MTAI, a transcriptional activator of breast cancer amplified sequence 3 . Proc Natl Acad Sci USA 2006, I 03(17):6670-5.

36. Jang KS, Paik SS, Chung H, Oh YH, Kong G: MTAI overexpression correlates significantly with tumor grade and angiogenesis in human breast cancers. Cancer Sci 2006, 97(5):374-9.

37. Yi C, Li X, Xu W, Chen A: Relationship between the expression of MTA-I gene and the metastasis and invasion in human osteosarcoma. J Huazhong Univ Sci Technolog Med Sci 2005, 25(4):445-7.

38. Hofer MD, Menke A, Genze F, Gierschik P, Giehl K: Expression of MTAI promotes motility and invasiveness of PANC-I pancreatic carcinoma cells. Br J Cancer 2004, 26;90(2):455-62.

39. Toh Y, Ohga T, Endo K, Adachi E, Kusumoto H, Haraguchi M, Okamura T, Nicolson GL: Expression of the metastasis-associated MTAI protein and its relationship to deacetylation of the histone $\mathbf{H 4}$ in esophageal squamous cell carcinomas. Int J Cancer 2004, I I 0(3):362-7.

\section{Pre-publication history}

The pre-publication history for this paper can be accessed here:

http://www.biomedcentral.com/1471-2407/8/304/pre pub 